\title{
Fomento de la lectura y escritura en otras lenguas en el ámbito universitario: experiencia didáctica con estudiantes del Northwest Cádiz Program'
}

\author{
Promoting Literacy Skills in Other Languages at the University Level: \\ Teaching Experience with Students of the Northwest Cádiz Program
}

\author{
ALICIA MARISCAL RÍOS \\ Universidad de Cádiz e Instituto de Lingüística Aplicada (ILA) \\ España \\ alicia.mariscal@uca.es
}

(Recibido: 24/II/2OI8; aceptado: 03/05/2019)

Resumen. Nuestra propuesta supone una iniciativa de aplicación docente en el ámbito de la lingüística aplicada a la enseñanza de lenguas para la mejora de las destrezas escritas en otras lenguas por parte de estudiantes universitarios. Se trata de una experiencia didáctica diseñada para estudiantes del Northwest Cádiz Program, dirigido a alumnos estadounidenses de tercer y cuarto ciclo - procedentes de las Universidades de Washington y Western Washington-, que visitan Cádiz con el fin de vivir una experiencia de inmersión tanto lingüística como cultural. El objetivo principal que perseguimos es mejorar el aprendizaje de lenguas extranjeras -Español como Lengua Extranjera (ELE), en este caso- mediante el empleo en el aula de una metodología basada en la exposición a materiales escritos a través de lecturas tanto en la lengua nativa de los aprendices como en su propia lengua materna, que fomenten el análisis contrastivo y la traducción, así como la escritura de textos académicos propios del ámbito universitario en la lengua meta.

Palabras clave: análisis contrastivo; exposición a materiales escritos; fomento de las destrezas escritas en estudiantes universitarios; lingüística aplicada a la enseñanza de lenguas.
Abstract. This paper explores a teaching methodology - within the frame of Linguistics Applied to Language Teaching - for the development of written skills in other languages by college students. This proposal has been designed as part of our teaching experience with students belonging to the Northwest Cádiz Program. Students from the Universities of Washington and Western Washington come to Cádiz to enjoy a language immersion experience. Written skills in Spanish as a Foreign Language (SFL) are encouraged by means of their exposure to printed texts in both English and Spanish. This methodology favours contrastive analysis, translation, and the writing of academic essays in the target language within the context of higher education.

Keywords: contrastive analysis; exposure to print; development of written skills by college students; Linguistics Applied to Language Teaching.

\footnotetext{
${ }^{\text {I }}$ Para citar este artículo: Mariscal Ríos, Alicia (2020). Fomento de la lectura y escritura en otras lenguas en el ámbito universitario: experiencia didáctica con estudiantes del Northwest Cádiz Program. Alabe 21. [www.revistaalabe.com]

DOI: IO.I5645/Alabe2O2O.2I.8
} 


\section{Introducción}

A lo largo de los años, han sido distintos los métodos y enfoques propuestos para la enseñanza de lenguas extranjeras, desde el método tradicional (o de gramática y traducción), que enfatizaba la importancia de la enseñanza de la gramática y el vocabulario -aunque este último descontextualizado en la mayoría de los casos-, hasta el enfoque comunicativo actual, promovido por el Marco Común Europeo de Referencia para las lenguas (MCER) y el Plan Curricular del Instituto Cervantes, que concede el máximo peso a las interacciones comunicativas. Una postura lamentablemente bastante extendida hoy en día consiste en rechazar sistemáticamente la enseñanza explícita de la gramática, lo que ha llevado a algunos a pecar de cierta "comunicativitis". Además de si se debe o no enseñar la gramática de una forma explícita, el debate ha girado muchas veces en torno a si los profesores deberían permitir o no la utilización de la lengua materna de los alumnos en el aula. En nuestro caso, consideramos que el uso de la lengua materna por parte del alumno y del profesor puede mejorar la comprensión de la lengua meta y la fluidez comunicativa en ambas lenguas, ya que entendemos que recurrir a la lengua materna forma parte de la competencia estratégica²; de ahí que en los últimos años haya tomado peso la defensa del translanguaging (García, 2009), que propicia el desarrollo del potencial comunicativo en ambas lenguas - tanto la materna como la lengua meta-, con la inclusión de actividades que mejoran las destrezas lingüísticas de los estudiantes y su competencia comunicativa bilingüe, en el marco de "su identidad mixta y biculturalidad" (Mariscal, 2OI7: 468). El análisis contrastivo de las lenguas implicadas posibilita, a su vez, su capacidad analítica, dado que permite comprobar similitudes, pero sobre todo diferencias, entre los dos sistemas lingüísticos y favorecer, de este modo, el aprendizaje.

En la lengua materna, el lenguaje oral se adquiere de forma intuitiva y natural por parte del niño a través de las experiencias lingüísticas que le proporciona su entorno más inmediato. Por el contrario, las destrezas escritas, es decir, la lectura y la escritura, requieren un aprendizaje explícito en la escuela. Con respecto a la escritura, para su dominio no basta con conocer la ortografía, el léxico y la gramática del idioma, sino que también necesitamos poseer ciertas competencias tanto sociolingüísticas como discursivas y pragmáticas, que nos permitan elaborar textos apropiados, de acuerdo con el contexto, el registro y las características de nuestro(s) interlocutor(es).

En el ámbito de la Universidad, nos encontramos en muchas ocasiones con una falta de competencia comunicativa escrita en los estudiantes, quienes son incapaces de escribir textos en lenguaje académico, ni siquiera en su lengua materna. El problema se agrava aún más para los estudiantes extranjeros, quienes cuentan con la dificultad añadida de tener que redactar trabajos propios del ámbito universitario en la lengua que están aprendiendo.

\footnotetext{
${ }^{2}$ Véase Canale y Swain (1980).
} 
Con el fin de tratar de remediar este problema, hemos diseñado - en el ámbito de la linguística aplicada a la enseñanza de lenguas- una propuesta didáctica para nuestros alumnos del Northwest Cádiz Program, con el fin de desarrollar sus destrezas escritas en español, sin olvidar en ningún caso el refuerzo simultáneo de las orales. Para ello, les acercamos a obras literarias escritas en español, para, a partir de su lectura y análisis crítico, favorecer la puesta de sus competencias en acción a través de la escritura de ensayos académicos.

Este enfoque contribuye, al mismo tiempo, a despertar su interés por la lectura de otras obras literarias y ello puede resultar sin duda muy enriquecedor para los aprendices de lenguas en contextos universitarios en general, porque les permite incrementar su vocabulario, así como mejorar su gramática y sus destrezas escritas. Sus conocimientos culturales y sus destrezas orales son a su vez impulsadas gracias al visionado de adaptaciones cinematográficas de los trabajos analizados y la realización de debates y exposiciones, de modo que sus aprendizajes sean más significativos y motivadores.

En los dos siguientes capítulos, explicaremos de forma resumida dos de los pilares fundamentales de nuestra propuesta: el análisis contrastivo, por un lado, y la importancia de la exposición a materiales escritos en el desarrollo de las destrezas escritas, por otro.

\section{Papel del análisis contrastivo en la enseñanza de lenguas}

El análisis contrastivo surge en los años cuarenta del siglo XX (Fries, I945; Weinreich, I953, y Lado, 1957), en el seno de la lingüística aplicada a la enseñanza de lenguas, con el propósito inicial de comparar dos o más sistemas lingüísticos para detectar en qué modo las diferencias entre determinadas lenguas afectaban a su proceso de aprendizaje (Santos Gargallo, 1994). En un principio, se proponía "detectar los mecanismos que provocan errores entre los estudiantes” (Moure y Palacios, 1996: 69-70) y para ello se basaba en los siguientes procesos (Mariscal, 2OI7a): (I) describir cada sistema lingüístico que entraba en contacto (la lengua materna del aprendiz y la lengua meta); (2) seleccionar aquellas estructuras, el vocabulario y las reglas gramaticales que iban a ser objeto de análisis en cada lengua; (3) establecer las similitudes y diferencias que presentaban las lenguas contrastadas, y (4) predecir los errores que podían llegar a cometerse por interferencias entre los sistemas (errores interlingüísticos).

El problema surgió a la hora de dar cuenta de los errores cuya naturaleza no era propiamente interlingüística, sino que se debían al proceso de aprendizaje de la lengua meta per se (errores intralingü̈́sticos) y no a las interferencias de la lengua materna. Ello llevó a Wardaugh (I970) a proponer una distinción entre dos versiones del análisis contrastivo: una 'fuerte' (más descriptiva) y otra 'débil', relacionada con el análisis de errores, basada en el diagnóstico a partir de producciones reales de los aprendices. Creemos que el uso de informaciones basadas en el análisis contrastivo puede ser beneficioso en 
la enseñanza de lenguas, pues los conocimientos sobre la estructura de la lengua meta ayudan a prever posibles dificultades gramaticales, que precisan de una mayor atención en clase (Mariscal, 2OI7a).

Coincidimos, por tanto, con Fernández Pérez ( 1986 : $5^{\mathrm{I}-5^{2}}$ ) en que la lingüística contrastiva favorece el aprendizaje de otras lenguas, al aprovechar los conocimientos del idioma que se enseña y del que ya se domina para comprobar sus similitudes y diferencias $^{3}$, de ahí que nuestra propuesta didáctica se base precisamente en el análisis contrastivo - entre textos escritos en inglés (la lengua materna de nuestros estudiantes) y el español (su lengua meta)-.

A continuación, explicaremos brevemente otro de los ejes de nuestro trabajo: la exposición a materiales escritos para el fomento de las destrezas escritas de estudiantes universitarios que aprenden otras lenguas.

\section{La exposición a materiales escritos bilingües para el fomento de las destrezas es- critas de estudiantes universitarios}

En este artículo, continuamos en la línea de otros trabajos (cf. Mariscal, 2OI7a, 20I7b y 20I8b) en los que pretendemos destacar la importancia de la exposición a materiales escritos en el desarrollo de la lectura y escritura, aunque aquí nos centraremos en su influencia en el aprendizaje de lenguas entre estudiantes universitarios. Se ha comprobado que dicha exposición es esencial en el caso de los niños y constituye -junto al fomento de la lectura y la escritura en el ámbito familiar ${ }^{4}$ - uno de los factores ambientales más importantes del procesamiento ortográfico (Powell, 20I5), entendido como la capacidad para formar, almacenar y acceder a las representaciones ortográficas (Cunnigham y Stanovich, I993: 193).

Además de repercutir positivamente en el procesamiento ortográfico, según Acheson, Wells y MacDonald (2008), una mayor exposición a los textos escritos conlleva también ciertas mejoras desde un punto de vista psicolingüístico, por ejemplo en el procesamiento fonológico (Siegel, I993; Torgesen, Wagner y Rashotte, I994); las tareas de decisión léxica (Chateau y Jared, 2000); la comprensión lectora (Cipielewski y Stanovich, I992); el vocabulario (Frijters, Barron y Brunello, 2000), y la fluidez verbal (Stanovich y Cunningham, I992), entre otras. De hecho, en su estudio con 99 estudiantes universitarios, Acheson et al. (2008) encontraron una correlación entre la exposición a materiales escritos y los logros lectores de los sujetos analizados.

\footnotetext{
${ }^{3}$ Como ejemplo de la utilización del análisis contrastivo en la enseñanza de ELE, véanse Mariscal (2OI6 y 20I8a).

${ }^{4}$ Consúltense los trabajos de Teale y Sulzby (I986); Sénéchal, Lefevre y Daley (I998); Duursma et al. (2007); Oller, Pearson y Cobo-Lewis (2OO7); Pearson (20O7), y Niklas (2OI3). El papel de la familia puede ser decisivo en estos casos, bien para fomentar la lectura o bien para inhibirla.
} 
Nuestro propósito es contribuir a la potenciación de la lectura y la escritura entre los estudiantes universitarios que aprenden otras lenguas, mediante la realización de actividades en ambos idiomas y la exposición a diferentes tipos de textos, como los mencionados en Mariscal (20I8b), basados a su vez en el cuestionario de lectura y hábitos lectores (Reading and Leisure Habits Questionnaire) de Atkinson (2015), que permite recoger datos sobre el número de horas de exposición a la lectura de diferentes recursos por parte de los aprendices, tanto impresos como electrónicos. Entre los ítems del cuestionario se encuentran los libros de texto y el material propiamente escolar, pero también diversas lecturas procedentes de libros, revistas y periódicos - en papel y en formato electrónico- y textos asociados a las nuevas tecnologías, como mensajes en el teléfono móvil, correos electrónicos, blogs y tuits, redes sociales y otro material procedente de internet.

A partir de estos materiales, hemos diseñado una propuesta didáctica, que desarrollamos en el siguiente capítulo, dirigida a estudiantes universitarios americanos pertenecientes al Northwest Cádiz Program, que pretende el fomento de la lectura, tanto en su lengua materna (el inglés) como en la lengua meta (el español), y la escritura de textos en lenguaje académico propios de un contexto universitario.

\section{Propuesta didáctica para la mejora de las destrezas escritas en otras lenguas en el ámbito universitario}

Como ya mencionamos más arriba, nuestra propuesta consiste en la mejora del aprendizaje de lenguas a través de la utilización en el aula de materiales en la lengua materna del alumno y la lengua meta, que favorezcan su análisis contrastivo y la exposición a materiales escritos de diferente naturaleza -tanto impresos como digitales-. A continuación, describiremos la metodología que empleamos con estudiantes del Northwest Cádiz Program, procedentes de las Universidades de Washington y Western Washington, que aprenden español en la Universidad de Cádiz.

La asignatura que impartimos, denominada Spanish 303 , nos ofrece la posibilidad de mejorar las destrezas escritas (en inglés y en español) de nuestros alumnos, ya que se trata de un curso de composición de nivel avanzado, cuyo objetivo fundamental es desarrollar "las técnicas y estrategias de escritura necesarias para la creación de ensayos de crítica literaria y análisis cultural [...] y preparar al estudiante para enfrentarse con éxito a la escritura académica [...] de nivel universitario"5.

Para ello, en clase no se trabaja únicamente con textos literarios, sino también con artículos periodísticos y cualquier otro material relacionado con el tema, procedente de páginas web en ambas lenguas, lo cual les obliga a leer tanto en inglés como en español, a entender su contenido y sintetizar la información más importante, que, posteriormente, es convertida en ensayos de crítica literaria. Este proceso de composición conlleva diver-

5 Toda la información sobre esta asignatura puede consultarse en la página web de la Universidad de Washington (Spanish and Portuguese Studies): ‘https://spanport.washington.edu/espanol-303>. 
sas fases: desde la planificación inicial del texto a la edición de dos borradores, que, una vez revisados por el profesor, constituyen la versión definitiva. De hecho, en la evaluación se valora todo el proceso y no solo el resultado final. Una de las revisiones consiste en una tarea de peer editing ("revisión entre pares"), en la que los aprendices colaboran en la edición y crítica de los trabajos de sus compañeros.

Algunas de las ventajas que ofrece esta metodología son, entre otras, la puesta en práctica de aprendizajes significativos y colaborativos, más activos, autónomos y funcionales. Las comparación de fragmentos de las obras originales y sus correspondientes traducciones al inglés facilita asimismo la realización de actividades de corte contrastivo, que favorecen la explicación de la gramática y una visión más analítica de ambos sistemas, a la vez que ofrecen una fuente inagotable de conocimientos sobre los diferentes mecanismos de traducción y mejoran su vocabulario y el aprendizaje de expresiones idiomáticas que no son traducibles a la otra lengua. En este sentido, las actividades de fraseología contrastiva resultan de gran valor, dado que enriquecen los conocimientos lingüísticos y culturales de la lengua meta. También destacamos la creación colectiva de un blog, en el que cada alumno publica enlaces e informaciones curiosas y de interés sobre los contenidos aprendidos a lo largo del curso, a los que el resto de estudiantes puede recurrir a la hora de redactar sus ensayos, por lo que se trata de aprendizajes de tipo colaborativo.

En cuanto a las destrezas orales, estas son fomentadas simultáneamente mediante tareas consistentes en presentaciones orales y el visionado -bien en español o bien en versión original subtitulada ${ }^{6}$ - de adaptaciones cinematográficas de obras relacionadas con su trabajo de investigación, como Blindness (2008), para trabajar Ensayo sobre la ceguera (Saramago, I995), o La novia (2015) en el caso de Bodas de sangre (Lorca, I933).

Para comprobar si nuestra propuesta influía o no positivamente en los resultados de nuestro grupo, los alumnos realizaron un test de evaluación inicial, que consistía en la traducción de dos textos escritos en lenguaje literario - uno en español y otro en inglés-, de modo que se esperaba que realizasen tanto una traducción directa (a su lengua materna, es decir, el inglés) como inversa (a la lengua meta, en este caso el español). Con estas dos actividades pretendíamos comprobar principalmente su ortografía y vocabulario en ambas lenguas, su gramática y el empleo de los diferentes marcadores discursivos.

Una vez finalizado el curso, volvimos a aplicarles la misma prueba, aunque con textos diferentes, que incluían conceptos ya trabajados en clase. Los resultados muestran una mejoría de los resultados en la mayor parte de los alumnos, puesto que, tras la aplicación de esta experiencia didáctica con nuestros estudiantes estadounidenses, hemos observado: (I) menos errores - de tipo inter- e intralingüístico (Mariscal, 20I8a)- desde un punto de vista ortográfico, léxico y gramatical; (2) un aumento de la riqueza de su vocabulario y un menor empleo de palabras "comodín", como el verbo "tener", que es reemplazado por otros más específicos, como "presentar" y "manifiestar"; (3) una

\footnotetext{
${ }^{6}$ En este segundo caso, los alumnos realizan también una actividad de lectura.
} 
sintaxis más compleja, con el uso de más oraciones coordinadas y subordinadas, en vez de oraciones simples separadas por punto, y (4) el empleo de sinónimos para evitar las repeticiones, como "porque", "pues", "ya que", "puesto que" o "dado que" para traducir because.

\section{Conclusiones y algunas recomendaciones}

Aunque no hay duda de que el enfoque comunicativo puede ser útil para el desarrollo de las destrezas orales, las escritas hacen necesaria una enseñanza mucho más explícita en el aula, puesto que exigen un proceso continuo de perfeccionamiento y la puesta en acción de las competencias del alumno para llegar a redactar textos académicos de calidad en el ámbito de la Universidad.

La enseñanza de lenguas constituye una excelente oportunidad para que los estudiantes universitarios creen ensayos a partir de lecturas en ambos idiomas, que favorezcan la traducción y el análisis contrastivo entre su lengua materna y aquella que es objeto de aprendizaje. La utilización de diferentes recursos en ambos idiomas-ya sean en papel o en formato electrónico- permite al estudiante una mayor exposición a materiales escritos y el aprendizaje por descubrimiento, gracias a la búsqueda de información, su lectura y el posterior análisis y tratamiento de la información como fuente de creación de sus trabajos académicos.

Todo ello contribuye, sin duda, al impulso de sus destrezas metacognitivas para "aprender a aprender" de forma autónoma y ayuda, al mismo tiempo, a mejorar su ortografía, vocabulario y gramática, así como a potenciar un tratamiento más analítico y significativo de ambos sistemas. 


\section{Referencias bibliográficas}

-Acheson, D. J., Wells, J. B. y MacDonald, M. C. (2008). New and updated tests of print exposure and reading abilities in college students. Behavior Research Methods, 4O(I), 278289 .

-Atkinson, L. (20I5). A Longitudinal Investigation of the Social, Cognitive and Social Cognitive Predictors of Reading Comprehension. Tesis Doctoral inédita. University of Roehampton.

- Canale, M. y Swain, M. (1980). Theoretical bases of communicative approaches to second language teaching and testing. Applied Linguistics, I(I): I-47.

- Chateau, D. y Jared, D. (200o). Exposure to print and word recognition process. Memory and Cognition, 28, I43-I53.

- Cipielewski, J. y Stanovich K. E. (1992). Predicting growth in reading ability from children’s exposure to print. Journal of Experimental Child Psychology, 54, 74-89.

- Cunnigham, A. E. y Stanovich, K. E. (1993). Children’s Literacy Environments and Early Word Recognition Subskills. Reading and Writing, 5(2), I93-204.

- Duursma, E.; Romero-Contreras, S.; Szuber, A.; Proctor, P.; Snow, C.; August, D., y Calderón, M. (2007). The Role of Home Literacy and Language Environment on Bilinguals' English and Spanish Vocabulary Development. Applied Psycholinguistics, 28, I7 I-I9O.

- Fries, C. C. (1945). Teaching and Learning English as a Foreign Language. Michigan: The University of Michigan Press.

- Frijters, J. C., Barron, R. W. y Brunello, M. (200o). Direct and mediated influences of home literacy and literacy interest on prereaders' oral vocabulary and early written language skill. Journal of Educational Psychology, 92, 466-477.

- García, O. (2009). Bilingual Education in the 21st Century: A Global Perspective. Oxford: Blackwell.

- García Lorca, F. (I933). Bodas de sangre. Madrid: Cátedra.

- Grant, A., Wilson, A. M. y Gottardo, A. (2007). Exposure in Reading Skills of Postsecondary Students with and without Reading Disabilities. Exceptionality Education International, I7 (2), I75-194.

- Lado, R. (1957). Linguistics across Cultures: Applied Linguistics for Language Teachers.

Michigan: The University of Michigan Press. 
- Mariscal Ríos, A. (20I6). El nuevo rol de la interjección en la enseñanza de lenguas extranjeras: estudio contrastivo inglés-español. En Y. Morimoto, Ma V. Pavón y R. Santamaría (Eds.). La enseñanza de ELE centrada en el alumno (pp. 565-573). Getafe: ASELE.

- Mariscal Ríos, A. (20I7a). Análisis de errores ortográficos (inglés/español) en estudiantes de Educación Secundaria en una zona de contacto lingüístico: Gibraltar. Tesis doctoral inédita. Universidad de Cádiz.

- Mariscal Ríos, A. (20Izb). El papel de la ortografía en el aprendizaje de la lectura. En E. Jiménez Pérez (Coord.). CIVEL: Educación lectora (pp. I65-I72). Madrid: Síntesis.

- Mariscal Ríos, A. (20r8a). Propuesta de taxonomía para el análisis de errores ortográficos de aprendices de ELE con inglés como Li. En V. Gaviño Rodríguez y J. Marchena Domínguez (Eds.). Civilización, literatura y lengua españolas. Ciencia y docencia en el NW-Cádiz Program (1997-20I7) (pp. 2I3-223). Cádiz: Servicio de Publicaciones de la Universidad de Cádiz.

- Mariscal Ríos, A. (2018b): Influencia de la exposición a materiales escritos en el desarrollo de la lectoescritura. En Ma I. de Vicente-Yagüe y E. Jiménez Pérez (Eds.), Investigación e innovación en educación literaria (pp. I25-I3I). Madrid: Síntesis.

- Oller, D. K., Pearson, B. Z. y Cobo-Lewis, A. B. (2007). Profile Effects in Early Bilingual Language and Literacy. Applied Psycholinguistics, 28, I9I-23O.

- Pearson, B. Z. (2007). Social Factors in Childhood Bilingualism in the United States. Applied Psycholinguistics, 28, 399-4IO.

- Powell, D. (2015). The Challenges of Learning to Read and Write in English. Patoss Bulletin, 28(I), 2-6.

- Saramago, J. (20I5). Ensayo sobre la ceguera. Barcelona: Debolsillo.

- Sénéchal, M., Lefevre, J. A., Thomas, E. M. y Daley, K. E. (1998). Differential Effects of Home Literacy Experiences on the Development of Oral and Written Language". Reading Research Quarterly, 33(I), 96-II6.

- Siegel, L. (1993). Phonological processing deficits as the basis of a reading disability. Developmental Review: Phonological processes and learning disability, I3, 246-257.

- Stanovich, K. E. y Cunningham, A. E. (1992). Studying the consequences of literacy within a literate society: The cognitive correlates of print exposure. Memory and Cognition, 2O, $5^{\mathrm{I}-68 .}$ 
- Stanovich, K. E. y West, R. F. (1989). Exposure to Print and Orthographic Processing. Reading Research Quarterly, 24, 402-433.

- Teale, W. H. y Sulzby, E. (ig86). Emergent Literacy as a Perspective for Examining how Young Children Become Writers and Readers. En W. H. Teale y E. Sulzby (Eds.). Emergent literacy: Writing and reading (pp. 7-25). New Jersey: Ablex.

- Torgesen, J. K., Wagner, R. K. y Rashotte, C. A. (I994). Longitudinal studies of phonological processing and reading. Journal of Learning Disabilities, 27, 276-286.

- Weinreich, U. (1953). Languages in Contact. Findings and problems. The Hague: Mouton. 